J. Urol. Urogynäkol. AT 2019 • 26:92-97 https://doi.org/10.1007/s41972-019-00080-8 Online publiziert: 8. Oktober 2019 (c) Der/die Autor(en) 2019

\section{Sophina Bauer ${ }^{1}$ Lukas Lusuardi ${ }^{2}$}

'Universitätsklinikum für Urologie und Andrologie, Salzburger Landeskliniken, Salzburg, Österreich

${ }^{2}$ Department of Urology, Paracelsus Medical University Salzburg, Salzburg, Österreich

\title{
Therapie der überaktiven Blase
}

\section{Erweiterung der konservativen Therapie durch Etablierung der Kombinationstherapie}

\section{Einleitung}

Aus politischer Sicht ist die überaktive Blase (OAB) ein Symptomenkomplex mit zunehmender Prävalenz und ökonomischer Bedeutung. Die Betroffenen selbst leiden unter einer massiven Einschränkung ihrer Lebensqualität mit sozialer Isolation und häufig Depression. Oft gibt es für die OAB keine pauschale Heilung, da die Ursache nicht immer behoben werden kann. Nach einer genauen Diagnostik muss mit jedem Patienten und jeder Patientin individuell eine Therapie erarbeitet werden. Eine gute Kommunikation zwischen den Behandelnden und den Betroffenen stellt die Basis für einen Therapieerfolg dar. Darauf aufbauend kommt der verhaltensadaptierenden Therapie die größte Bedeutung zu. Oft muss zusätzlich eine medikamentöse Therapie erfolgen. Auch hier tritt eine Besserung oft erst nach mehrfachem Wechsel des Präparates ein. Seit kurzem wird von den internationalen Leitlinien die Kombinationstherapie von Anticholinergika mit Beta-3-RezeptorAgonisten empfohlen, was eine Erweiterung der konservativen Therapie bedeutet. Werden invasivere Therapieformen notwendig, sollten diese auf die Betroffenen abgestimmt sein und deren persönlichen Therapiewunsch entsprechen. Dieser Herausforderung müssen sich die Ärztinnen und Ärzte stellen, die eine erfolgreiche Therapie der überaktiven Blase anstreben.

\section{Erstlinientherapie: Verhaltensanpassung und Miktionstraining}

Am Beginn der Therapie steht eine exakte Aufklärung der Patientinnen und Patienten über ihr Leiden sowie den Therapieablauf im Vordergrund. Bereits durch das Führen des Miktionstagebuches kann den Betroffenen geholfen werden, sich ein Bild des Ausmaßes ihrer Erkrankung zu machen. Oftmals divergiert die subjektive Belastungssituation von der tatsächlichen Miktionsfrequenz. Auch können direkte Zusammenhänge der überaktiven Blase mit dem Trinkverhalten besprochen werden. Ziel ist eine gleichmäßige Hydrierung, die sich in der Senkung des spezifischen Uringewichtes zeigt $(<1015 \mathrm{~g} / \mathrm{l})$. Somit können zum einen rezidivierende Infekte vermieden und zum anderen die OAB-Symptomatik bereits gebessert werden [1].

Eine gute, empathische Kommunikation zwischen dem Behandelnden und der Patientin bzw. dem Patienten stellt die Basis des Therapieerfolgs beim Miktionstraining dar. In weiterer Folge ist eine adäquate Beckenbodenschulung unerlässlich, welche die willkürliche Aktivierung der richtigen Muskelgruppen stärkt bzw. ermöglicht. Unter Anleitung lernen die Patientinnen und Patienten, den imperativen Harndrang zu unterdrücken und die Miktionsfrequenz zu reduzieren („bladder drill“). Weiters muss individuell der Entleerungsrhythmus an die individuelle Blasenkapazität angepasst werden, um Dranginkontinenzepisoden zu reduzieren. Auch hier kann ein gut geführtes Miktionsprotokoll als Vorlage zur Therapieplanung dienen. Als Ergänzung zum Beckenbodentraining wird eine Intensivierung mit Biofeedback und Elektrostimulation als Kombinationstherapie von den S2kLeitlinien der DGGG empfohlen [2].

\section{Zweitlinientherapie: Pharmakotherapie}

In vielen Fällen ist neben der Verhaltensanpassung auch eine medikamentöse Therapie der überaktiven Blase notwendig. Den größten Pfeiler stellt seit vielen Jahren die antimuskarinerge Therapie mit Anticholinergika da. Sie wirken über eine kompetitive Hemmung der muskarinergen Rezeptoren M2 und M3 im Urothel sowie in den suburothelialen Myofibroblasten. Die nun verhinderte Kontraktion der Blasenmuskulatur über Azetylcholin führt zu einer Verbesserung der Blasenkapazität und zum Rückgang der OAB-Symptomatik [1]. Trotz des breiten Einsatzes der Anticholinergika ist die Absetzrate bereits nach wenigen Monaten sehr hoch. Zum einen ist dies auf die anticholinergen Nebenwirkungen wie Mundtrockenheit, Verstopfung, Harnretention und Sehstörungen zurückzuführen. Zum anderen tritt die Wirkung erst nach mehreren Wochen regelmäßiger Einnahme ein und erzielt nicht immer den gewünschten Erfolg [1]. Häufig müssen die Betroffenen über mehrere Monate verschiedene Anticholinergika einnehmen, bis sie mit dem Wirk- und Nebenwirkungsprofil zufrieden sind.

Die Gruppe der Anticholinergika umfasst mehrere Substanzen, die eine vergleichbare Effektivität zeigen. Oxybutynin ist das bekannteste Anticholinergi- 
kum, welches sowohl in der Therapie der neurogenen Blase als auch in der Kinderurologie einen wichtigen Stellenwert hat. Neben der oralen Darreichungsform gibt es auch die transdermale und die intravesikale Applikation. Das Auftreten von Mundtrockenheit ist jedoch höherim Vergleich zu anderen Antimuskarinika wie Tolterodin [3]. Die Substanzen unterscheiden sich hauptsächlich in ihrer Selektivität bezüglich der Muskarinrezeptoren. Bis auf Trospiumchlorid handelt es sich um tertiäre Amine, die über die Leber und das Zytochrom CYP3A4 metabolisiert werden, was zu Wechselwirkungen mit vielen anderen Medikamenten und Nahrungsmitteln führen kann. Trospiumchlorid ist ein quartäres Ammoniumderivat, welches hydrophil ist und ausschließlich über die Niere eliminiert wird. Des Weiteren kann es aufgrund seiner Hydrophilie die Blut-Hirn-Schranke nicht passieren und führt somit nicht $\mathrm{zu}$ zentralen Nebenwirkungen. Dies ist insbesondere in der Behandlung des geriatrischen Patientenklientels von Bedeutung (s. unten).

Der Therapieerfolg sollte nach 4-6 Wochen mittels Miktionstagebuch evaluiert werden, ggf. sollte dann eine Therapieanpassung erfolgen. $\mathrm{Zu}$ berücksichtigen sind die Kontraindikationen wie ein unbehandeltes Engwinkelglaukom, mechanische Stenosen des Gastrointestinaltraktes, tachykarde Herzrhythmusstörungen sowie die Myasthenia gravis [4]. Auch eine Restharnbildung über $100 \mathrm{ml}$ ist als kritisch zu werten, da es unter einer anticholinergen Therapie zu einem Harnverhalt kommen kann.

Seit 2012 wurde ein weiteres Medikament von der FDA („Food and Drug Administration") zur Therapie der überaktiven Blase zugelassen. Mirabegron ist ein Beta-3-Adrenozeptor-Agonist, der durch Bindung an den Beta-3-Adrenozeptor die Azetylcholinfreisetzung herunterreguliert und somit ebenfalls die Blasenfüllung verbessert und die Drangsymptomatik verringert.

Hervorzuheben ist, dass Mirabegron im Gegensatz zur Gruppe der Antimuskarinika keinen Einfluss auf die Entleerungsphase der Miktion hat und somit breiter eingesetzt werden kann [5]. Insgesamt wurde in mehreren Studien ge- zeigt, dass Mirabegron die Miktionsfrequenz, die Dranginkontinenzrate und die Drangsymptomatik im Vergleich zu Plazebo senkt [5].

$\mathrm{Zu}$ den häufigsten Nebenwirkungen zählen Kopfschmerz und Nasopharyngitis.

Es besteht eine Kontraindikation für den Einsatz bei Patienten und Patientinnen mit unkontrollierter schwerer Hypertension ( $R R$ systolisch $\geq 180 \mathrm{~mm} \mathrm{Hg}$ / diastolisch $\geq 110 \mathrm{~mm} \mathrm{Hg}$ ), und bei Patienten und Patientinnen mit Neigung zu Vorhofflimmern ist Vorsicht geboten [4].

Seit kurzem liegen die Daten zum Einsatz einer Kombinationstherapie aus Anticholinergika (Solifenacin) und dem Beta-3-Adrenozeptor-Agonisten Mirabegron vor. Ziel ist, eine höhere Ansprechrate bei jenen Patientinnen und Patienten zu erreichen, bei welchen die Monotherapie mit einem der Präparate frustran verlief. In 3 groß angelegten Studien („SYMPHONY trial“, „BESIDE trial“ und "SYNERGY trial“) werden die Sicherheit und Effizienz des gemeinsamen Einsatzes von Solifenacin und Mirabegron analysiert [1]. Die SYMPHONY-Studie zeigte in den Gruppen der Kombinationstherapie ein erhöhtes Miktionsvolumen, einen Rückgang der Miktionsfrequenz, der Drangepisoden sowie der Inkontinenzvorfälle. Das Nebenwirkungsprofil war vergleichbar mit dem der Einzeltherapie ohne Erhöhung des Restharns oder des Blutdrucks. Alleine die Rate an Verstopfung war leicht erhöht $[1,6]$. Die BESIDE-Studie untersuchte Patientinnen und Patienten mit einem initial schlechten Ansprechen bei Monotherapie mit Solifenacin. Auch hier zeigten sich in der Kombinationstherapie ein deutlicher Rückgang der Miktionsfrequenz sowie eine Besserung der Lebensqualität ohne signifikante $\mathrm{Zu}$ nahme der Nebenwirkungen. In Bezug auf die Dranginkontinenz war die Kombinationstherapie der Monotherapie mit Solifenacin nicht unterlegen.

Die SYNERGY-Studie zeigte eine Überlegenheit der Kombinationstherapie in Hinblick auf die Besserung der OAB-Symptomatik, jedoch keine Überlegenheit im Rückgang der Dranginkontinenzepisoden im Vergleich $\mathrm{zu}$ Mirabegron alleine.
Insgesamt kann der Einsatz einer Kombinationstherapie aus Solifenacin und Mirabegron nach frustraner Monotherapie als effektiv und sicher angesehen werden und den Betroffenen eine invasivere Therapie ersparen bzw. diese verzögern. So hielt die Kombinationstherapie bereits Einzug in die amerikanischen Leitlinien der AUA und SUFU [1, 6].

Auch weitere Substanzen wie trizyklische Antidepressiva wurden früher zur Therapie der OAB eingesetzt, sind jedoch heute nur noch im off label use im Einsatz.

Die Alpha-1-Blocker, wie Tamsulosin, Doxazosin oder Alfuzosin, haben zwar keine direkte Wirkung auf die überaktive Blase, senken jedoch bei Prostatahyperplasie den Auslasswiderstand und können somit Symptome bei Männern mit Obstruktion lindern. Bei der Frau stellt ihr Einsatz einen off label use dar [4].

Neben weiteren pharmakologischen Substanzen, die nur wenig Bedeutung in der Therapie der OAB haben, stellt die lokale Östrogenisierung bei der Frau eine wichtige Maßnahme dar, die früh eingesetzt werden sollte. Eine Cochrane-Analyse zeigte $57 \%$ subjektive Verbesserung der Dranginkontinenz durch alleinige Östrogentherapie im Gegensatz zu $27 \%$ bei Plazebo [7].

\section{Drittlinie: Minimalinvasive Verfahren}

Wenn trotz mehrfacher medikamentöser Therapie und Miktionstraining kein Therapieerfolg erzielt werden kann, stehen mehrere minimalinvasive Verfahren zur Linderung der OAB zur Verfügung.

\section{Glykosaminoglykanersatztherapie}

Das Urothel wird durch eine Schicht von Glykosaminoglykanen (GAG) wie Hyaluronsäure und Chondroitinsulfat ausgekleidet, die es vor einer Irritation durch Kaliumionen im Harn schützt. Wenn die GAG-Schicht brüchig wird, kommt es zu einer Reizung der Blasenwand mit folglich Blasenschmerzen und Drangsymptomatik.

Durch eine 6- bis 8-wöchige Instillationstherapie (1-mal/Woche) soll die 
GAG-Schicht wieder aufgebaut und stabilisiert werden. Diese Therapie wird für rezidivierende Harnwegsinfekte verbreitet eingesetzt, jedoch stellt sie in der Therapie der $\mathrm{OAB}$ nur ein Reserveverfahren dar [4].

\section{Onabotulinumtoxin A}

Nach Versagen der medikamentösen Therapie und hohem Leidensdruck stellt die intravesikale Applikation von Botulinumtoxin eine effektive Therapieoption dar. Das Neurotoxin wird in einer Dosierung von 100 Einheiten in $\mathrm{NaCl}$ verdünnt auf 10-30 Injektionen in die Blasenwand injiziert. Dadurch erfolgt eine lokale reversible Denervierung durch Hemmung der Ausschüttung von Azetylcholin in den synaptischen Spalt. Innerhalb weniger Tage tritt die Wirkung ein und hält für 9-12 Monate an. In einer großen multizentrischen Phase-3-Studie konnte eine signifikante Reduktion der Inkontinenzepisoden im Vergleich zu Plazebo gezeigt werden. $22,9 \%$ erreichten sogar eine komplette Kontinenz durch die Therapie. $\mathrm{Zu}$ den Nebenwirkungen zählen Dysurie, Harnwegsinfekte und Harnretention. Vor dem Einsatz von Botulinumtoxin muss in jedem Fall die Bereitschaft der Patientinnen und Patienten zum Einmalkatheterismus besprochen werden, da eine behandlungsbedürftige Retention in ca. $6 \%$ der Fälle auftritt.

Auch wiederholte Botulinumtoxininjektionen haben ihre Langzeiteffektivität bewiesen [8].

\section{Sakrale Neuromodulation}

Die sakrale Neuromodulation erfolgt über eine gezielte Stimulation der sakralen Nervenwurzel S3 über fluoroskopisch gelegte Elektroden. Trotz intensiver Erforschung der seit 1981 von Tanagho et al. beschriebenen Therapieform ist der Effekt der Neuromodulation bis heute nicht vollständig geklärt [9]. Vermutlich wird über die Modulation der spinalen und supraspinalen afferenten Nervenfasern eine Reduktion der OABSymptomatik erreicht [10].

In der ersten Phase wird der mögliche Therapieerfolg über eine Testpha-

J. Urol. Urogynäkol. AT 2019 -26:92-97 https://doi.org/10.1007/s41972-019-00080-8

(c) Der/die Autor(en) 2019

\section{S. Bauer $\cdot$ L. Lusuardi} Therapie der überaktiven Blase. Erweiterung der konservativen
Therapie durch Etablierung der Kombinationstherapie

\section{Zusammenfassung}

Der Symptomenkomplex der überaktiven Blase (OAB) führt bei den Betroffenen zu einer ausgeprägten Minderung der Lebensqualität, die oftmals mit sozialer Isolation verbunden ist.

Nach einer genauen Diagnostik muss mit jedem Patienten und jeder Patientin individuell eine Therapie erarbeitet werden. Eine gute Kommunikation zwischen den Behandelnden und den Betroffenen stellt die Basis für den Therapieerfolg dar.

Die Erstlinientherapie besteht aus Adaptation des Verhaltens und der Trinkmenge sowie dem Blasentraining. Begleitend soll dazu ein Beckenbodentraining erfolgen. Führen diese Maßnahmen nicht zur erwünschten Besserung, kommen Anticholinergika zum Einsatz. Da es zu einer hohen Absetzrate aufgrund von Unverträglichkeiten bzw. Nebenwirkungen kommt, gewann in den letzten Jahren der neu etablierte Beta-3-RezeptorAgonist Mirabegron an Bedeutung. Seit 2019 ist auch die Kombinationstherapie der beiden oben genannten Substanzgruppen erstmals in den amerikanischen Leitlinien verankert und ermöglicht so einen breiteren Einsatz der oralen Therapie. Wenn alle konservativen Therapieoptionen ausgeschöpft sind, kommen minimalinvasive Verfahren wie die intravesikale Applikation von Botulinumtoxin oder die sakrale Neuromodulation zum Einsatz. Als Ultima Ratio bleibt bei immenser Lebensqualitätseinschränkung die Blasenaugmentation oder Harnableitung. Die Therapie der OAB bei älteren Personen stellt durch Polymedikation und Multimorbidität eine besondere Herausforderung dar.

\section{Schlüsselwörter}

Überaktive Blase - Biofeedback-Therapie . Kombinationstherapie - Anticholinergika . Beta-3-Agonisten

\section{Treatment of overactive bladder. Expanding on conservative treatment by establishing combination therapy}

\section{Abstract}

The symptom complex of overactive bladder $(\mathrm{OAB})$ leads to a considerable loss in quality of life for the patient concerned. The consequences are often depression and social isolation. Accurate diagnostics form a solid foundation for individual therapy planning. Secondly, good and empathetic communication between the practitioner and the patient is essential for therapeutic success.

First-line therapy includes a change of bladder and drinking behaviour combined with pelvic floor muscle training. Secondline therapy consists of medical treatment with antimuscarinics and beta- 3 agonists. Due to a high rate of side effects and/or lack of efficacy, compliance and adherence rates with antimuscarinics are low. According to an update issued by the American Urological Association, a combination therapy of anti- muscarinics and beta-3 agonists (solifenacin and mirabegron) may be considered in patients refractory to monotherapy. When pharmacotherapy fails, minimally invasive treatments like botulinum toxin A injection to the bladder or sacral neuromodulation are safe options to treat OAB symptoms. More extensive surgical treatments like bladder augmentation or urinary diversion should only be considered as last-line options in patients with massive complaints. The treatment of $O A B$ in the elderly remains a challenge due to multimorbidity and polypharmacotherapy.

Keywords

Overactive bladder - Biofeedbacktherapy . Combination therapy - Anticholinergics . Beta-3 agonists 
se evaluiert. In einem Eingriff in Allgemeinanästhesie werden unter fluoroskopischer Führung die sakralen Neuroforamina S3 punktiert und Elektroden an die Nervenwurzel S3 über SeldingerTechnik platziert. Diese werden über die Haut ausgeleitet und zur Testung über ein tragbares Aggregat über 1-6 Wochen stimuliert. In dieser Zeit wird das Ansprechen ambulant evaluiert. Wenn es zu einer $>50$ \%igen Reduktion der OABSymptomatik kommt, wird der Schrittmacher (IPG) in einem weiteren kleinen operativen Verfahren in eine subkutane Hauttasche glutäal implantiert. Verläuft die Testphase frustran, werden die Elektroden wieder operativ entfernt und andere Therapieoptionen evaluiert. Bis vor kurzem mussten Patientinnen und $\mathrm{Pa}$ tienten mit anatomischen Besonderheiten wie engen Neuroforamina oder Spina bifida von dieser Therapieform ausgeschlossen werden. Durch die Implementierung einer CT-gezielten Elektrodenplatzierung können nun auch Patientinnen und Patienten mit herausfordernder Anatomie eine sakrale Neuromodulation erhalten [11].

Voraussetzung für den dauerhaften Erfolg der Therapie sind eine hohe Compliance der Patienten sowie eine dauerhafte Anbindung an ein Zentrum, das die
Langzeitbetreuung der sakralen Neuromodulation übernehmen kann.

$\mathrm{Zu}$ den Risiken zählen die gehäuften operativen Revisionen bei Batteriewechsel (alle 3-5 Jahre), Elektrodendislokation (16\%), lokale Infektionen (2-5\%) und Verlust der therapeutischen Wirksamkeit (13\%; [10]).

Die bis heute eingesetzten Elektroden haben den Nachteil, dass sie nicht MRTtauglich sind und die Patienten bis auf Schädelaufnahmen keinen MRT-Untersuchungen unterzogen werden dürfen. Neuere MRT-taugliche Elektroden sollen bald auf den Markt kommen.

Generell wird die sakrale Neuromodulation heute für ein breites Spektrum der Blasen- und Darmentleerungsstörungen im neurogenen sowie nichtneurogenen Bereich eingesetzt. Wichtig ist die gute und gründliche Selektion der Patientinnen und Patienten, um langfristige Therapieerfolge zu erzielen.

\section{Ultima Ratio: Augmentation bzw. Harnableitung}

Eine Blasenaugmentation bzw. Zystektomie mit Neoblase oder anderweitiger Harnableitung stellt eine hochinvasive Therapieform dar und sollte ausschließlich in Einzelfällen als Ultima Ratio zum Einsatz kommen [4].

\section{OAB bei Patientinnen und Patienten über 65}

Mit zunehmendem Alter steigt die Prävalenz der OAB. So sind bis zu $20 \%$ der 65-Jährigen und bis zu $40 \%$ der 75-Jährigen von Symptomen der überaktiven Blase betroffen [1]. Die Behandlung der älteren Bevölkerung stellt eine besondere Herausforderung dar, da mehrere Faktoren bedacht werden müssen: Zum einen bestehen neben der überaktiven Blase häufig andere Komorbiditäten wie Obstipation oder kognitive Einschränkungen. Zum anderen nehmen viele Patientinnen und Patienten bereits mehrere Medikamente ein, die Interaktionen verursachen können. Drittens kann eine überaktive Blase durch medikamentöse Wechselwirkungen ausgelöst oder verstärkt werden. Besonders eine bestehende Stuhlinkontinenz oder eine chronische Obstipation können zu einer Dranginkontinenz führen [4].

\section{Alter und Anticholinergika}

Das Nebenwirkungsprofil der Anticholinergika stellt besonders im Alter eine Herausforderung dar. Gedächtnisstörungen, Verwirrtheit, Unruhe und Delir können eine vorbestehende Demenz deutlich verschlechtern bzw. eine beginnende

Hier steht eine Anzeige. 
Demenz demaskieren [8]. Das Anticholinergikum Trospiumchlorid hat hier ein Alleinstellungsmerkmal, da es durch seine Hydrophilie die Blut-Hirn-Schranke nicht passiert und somit keine ZNS-assoziierten Nebenwirkungen zu erwarten sind.

\section{Alter, Polymedikation und Inkontinenz}

Mit steigendem Alter kommt es zu einer Zunahme der chronischen Erkrankungen und folglich Polymedikation. Viele der häufig verschriebenen Medikamente steigern die Aktivität der Blase. Hierzu gehören u. a. Alpha-1-Blocker, ACEHemmer, NSAR, Kalziumkanalblocker [4]. Falls bereits eine Neigung zur überaktiven Blase vorbesteht, kann es durch den Einsatz solcher Präparate zu deren Verstärkung und Symptomen wie einer Dranginkontinenz kommen. Präparate mit einem diabetogenen Potenzial wie Statine, Betablocker, Glukokortikoide oder Thiaziddiuretika mindern die zentrale Hemmung des Kortex in der Steuerung der Blasenfunktion und führen ebenfalls zur einer Erhöhung der Blasenaktivität $[4,12]$. Eine genaue $\mathrm{Me}$ dikamentenanamnese sowie eine Umstellung der Medikation können bereits $\mathrm{zu}$ einer Besserung der $\mathrm{OAB}$ führen.

Des Weiteren muss bei geriatrischen Patienten mit Polymedikation die anticholinerge Last durch andere Medikamente bedacht und ggf. berechnet werden, bevor eine weitere anticholinerge Medikation begonnen wird. Ist diese bereits hoch, muss das weitere Anticholinergikum langsam auftitriert werden, um ein anticholinerges Syndrom zu verhindern. Erste Anzeichen einer Überbelastung mit Anticholinergika sind Benommenheit, Schwäche sowie Mundtrockenheit. Bei stärkerer Ausprägung kommt es auch zu Agitiertheit, Unruhe, Verwirrung und Gedächtnisstörungen. Peripher zeigen sich Obstipation, Tachykardie, Mydriasis sowie eine Harnretention. Ein schweres anticholinerges Syndrom ruft Halluzinationen, Ataxie und Krampfanfälle hervor. Weiters kommt es zu Schluckund Sprechstörungen, Arrhythmie und Harnverhalt. Im schlimmsten Fall kön- nen Koma und Schock bzw. peripher ein paralytischer Ileus auftreten [4]. Eine frühe Berechnung der anticholinergen Last beim geriatrischen Patienten kann diese Komplikationen im Voraus sicher verhindern.

Ein weiterer Aspekt der Polymedikation ist die vorwiegend hepatische $\mathrm{Me}$ tabolisierung der Anticholinergika über das Zytochrom CYP3A4, welches durch andere Induktoren (Johanniskraut, Glukokortikoide usw.) oder Hemmer (Diclofenac, Koffein usw.) zu ausgeprägten Wechselwirkungen führen kann.

Auch hier spielt Trospiumchlorid eine besondere Rolle. Da es ausschließlich renal eliminiert wird, kommt es zu keiner Interaktion mit CYP3A4. Bei reduzierter Nierenfunktion muss jedoch die Dosis von Trospiumchlorid angepasst werden [4].

\section{Alter und Aktivität}

Blasenentleerungsstörungen haben einen elementaren Einfluss auf die Lebensqualität. Die Auswirkungen eines sozialen Rückzugs durch Inkontinenz dürfen nicht unterschätzt werden. Während eine erhöhte körperliche Aktivität die Inkontinenz bessern kann, kann eine Isolation durch Inkontinenz zu einer Verschlechterung der kognitiven Leistungen führen [4]

\section{Therapie der überaktiven Blase im Alter}

Die gesamtheitliche Betrachtung der älteren Patientinnen und Patienten mit überaktiver Blase ist die Voraussetzung für einen Therapieerfolg. (Nicht-)medikamentöse Auslöser der Inkontinenz, soziale Folgen sowie Polymedikation müssen vor dem Beginn einer medikamentösen Therapieeinstellung bedacht werden.

Unter den Anticholinergika ist Trospiumchlorid für die geriatrischen Patientinnen und Patienten durch seine renale Elimination sowie die fehlende ZNSGängigkeit das am besten verträgliche Präparat.

Studien zur Sicherheit von Mirabegron zeigten eine vergleichbare Sicherheit des Präparates bei älteren Patientin- nen und Patienten wie in der jüngeren Bevölkerung. Zu bedenken ist jedoch die Sicherstellung eines guten Blutdruckprofils vor Beginn der Therapie.

Eine weitere Möglichkeit, die anticholinerge Last bei älteren Patientinnen und Patienten niedrig zu halten, ist die Kombinationstherapie aus einem niedrigdosierten Anticholinergikum mit Mirabegron. Die BESIDE-Studie zeigte einen guten Effekt der Kombinationstherapie (Solifenacin und Mirabegron) auch in der älteren Bevölkerung ohne relevante Zunahme der Nebenwirkungen [1, 2, 6].

Bei den invasiveren Therapieansätzen wie Botulinumtoxin oder der sakralen Neuromodulation sollten die kognitiven und körperlichen Voraussetzungen für die jeweilige Therapie vorher bedacht und mit den Betroffenen und ggf. Angehörigen besprochen werden. Das Risiko einer mehrfachen Narkosebelastung oder die Notwendigkeit einer vorübergehenden Harnableitung bzw. Selbstkatheterismus müssen dem Nutzen der Besserung der OAB-Symptomatik gegenübergestellt werden.

\section{Fazit für die Praxis}

Eine medikamentöse Therapie der
überaktiven Blase sollte nie ohne ein
Miktionstraining und eine entspre-
chende Aufklärung der Betroffenen
über Verhaltensadaptation erfolgen.
Zusätzlich zum Beckenbodentraining
ist ein Biofeedback sinnvoll.
Die anticholinerge Therapie wurde
vor einigen Jahren um den Beta-3-
Agonisten Mirabegron erweitert. Seit
heuer hat nun auch eine Kombina-
tionstherapie der beiden Gruppen
in den Leitlinien Einzug gefunden.
Dadurch kann Patienten nach initia-
lem Therapieversagen länger eine
konservative Therapie angeboten
werden.
Ältere Patienten stellen auch in der
Therapie der OAB eine Herausforde-
rung dar, da die anticholinerge Last
durch Polymedikation und Multimor-
bidität zu erheblichen Komplikati-
onen führen kann. Auch hier stellt
die Kombinationstherapie einen neu-
en Behandlungszweig dar, der die
anticholinerge Last verringert und


die Lebensqualität ohne relevante Änderung des Nebenwirkungsprofils erhöht.

\section{Korrespondenzadresse}

Dr. Sophina Bauer
Universitätsklinikum für
Urologie und Andrologie,
Salzburger Landeskliniken
Müllner Hauptstraße 48,
5020 Salzburg, Österreich
s.bauer@salk.at

Funding. Open access funding provided by Paracelsus Medical University.

\section{Einhaltung ethischer Richtlinien}

Interessenkonflikt. S. Bauer und L. Lusuardi geben an, dass kein Interessenkonflikt besteht.

Für diesen Beitrag wurden von der Autorin keine Studien an Menschen oder Tieren durchgeführt Für die aufgeführten Studien gelten die jeweils dort angegebenen ethischen Richtlinien.

Open Access. Dieser Artikel wird unter der Creative Commons Namensnennung 4.0 International Lizenz (http://creativecommons.org/licenses/by/4.0/deed. de) veröffentlicht, welche die Nutzung, Vervielfältigung, Bearbeitung, Verbreitung und Wiedergabe in jeglichem Medium und Format erlaubt, sofern Sie den/die ursprünglichen Autor(en) und die Quelle ordnungsgemäß nennen, einen Link zur Creative Commons Lizenz beifügen und angeben, ob Änderungen vorgenommen wurden.

\section{Literatur}

1. El-Zawahry A (2019) Combination pharmacotherapy for treament of overactive bladder $(\mathrm{OAB})$. Curr Urol Rep 20:33

2. Leitlinie: Die Überaktive Blase. Deutsche GesellschaftfürGynäkologie und Geburtshilfee. V. AWMF online (2010). https://www.awmf.org/leitlinien/ detail/ll/015-007.html.Zugegriffen:30.09.2019

3. Burkhard FC et al (2018) EAU quidelines on urinary incontinence in adults. European Association of Urology. Eur Urol 73(4):596-609. https://doi.org/ 10.1016/j.eururo.2017.12.031

4. Wiedemann A et al (2016) Überaktive Blase: Wissenswertes zu Anamnese, Diagnostik und Therapie in der Praxis. Diagnostik und Therapie in der Praxis. Deutscher Ärzteverlag, Köln

5. Chapple CR, Siddiqui E (2016) Mirabegron for the treatment of overactive bladder: a review of efficacy, safety, tolerability with a focus on male, elderly and antimuscarinic poor responder populations and patients with OAB in Asia. Expert Rev Clin Pharmacol. https://doi.org/10.1080/ 17512433.2017.1275570

6. Lightner DJ et al (2019) Diagnosis and treatment of overactive bladder (non neurogenic) in adults: AUA/SUFU guideline amendment.
JUrol 202(3):558-563. https://doi.org/10.1097/JU. 0000000000000309

7. Moehrer B, Hextall A, Jackson S (2013) Oestrogens for urinary incontinence in women. Cochrane Database Syst Rev (2):CD001405

8. Marinkovic SP (2019) New technologies in the management of overactive bladder: current research and future prospects. Ther Adv Urol. https://doi.org/10.1177/1756287219844669

9. Schmidt RA, Bruschini H, Tanagho EA (1978) Sacral root stimulation in controlled micturition. Peripheral somatic neurotomy and stimulated voiding. Invest Urol 17:130-134

10. Girtner F et al (2019) Sakrale Neuromodulation bei unter- und überaktivem Detrusor - quo vadis? Prinzipien und Entwicklungen. Urologe

11. Meissnitzer T et al (2015) CT-guided lead placement for selective sacral neuromodulation to treat lower urinary tract dysfunctions. AJR Am J Roentgenol 205(5):1139-1142

12. Yamaguchi $C$ et al (2007) Overactive bladder in diabetes: a peripheral or central mechanism? Neurourol Urodyn 26(6):807-813

Hinweis des Verlags. Der Verlag bleibt in Hinblick auf geografische Zuordnungen und Gebietsbezeichnungen in veröffentlichten Karten und Institutsadressen neutral.

\section{Kulturell-religiöse Aspekte in der Inkontinenz-Therapie}

In Österreich leben immer mehr Menschen mit unterschiedlichem kulturellem Hintergrund. Muslime bilden eine große Gruppe an Menschen, die auch Probleme mit der Kontinenz haben können. Sprachbarrieren sowie religiöse oder kulturelle Gepflogenheiten können Einfluss auf die erfolgreiche Behandlung und Versorgung von Patienten haben. Dies kann die Kommunikation zwischen Arzt und Patient bzw. Pflegeperson und Patient erschweren, zu Missverständnissen führen und damit den Aufbau einer vertrauensvollen Beziehung verhindern. Daher ist es hilfreich, Kenntnisse über die spezifischen Bedürfnisse dieser Patienten zu haben, um sie optimal versorgen zu können. Viele Betroffene wiederum sind unsicher und brauchen gezielte Informationen sowie ihren Lebensgewohnheiten angepasste Hilfs- und Lösungsmöglichkeiten.

Die MKÖ hat sich in Zusammenarbeit mit OA Dr. Kadir Tosun, stv. Referent des „Referat für Interkulturelle Zusammenarbeit und Integration" der Ärztekammer für Wien und Urologe am Krankenhaus Korneuburg, sowie in Abstimmung mit der Islamischen Glaubensgemeinschaft (IGGÖ) der Thematik der kultursensiblen Betreuung angenommen und folgende Materialien entwickelt:

Info-Folder für medizinisches und pflegendes Fachpublikum, der einen Überblick über die wichtigsten Aspekte im Umgang mit muslimischen Patienten mit Harnund/oder Stuhlinkontinenz enthält. Der Info-Folder umfasst Themen wie die Kommunikation, Ausübung religiöser Pflichten, Intimsphäre \& Scham etc.

Patienten-Folder in türksicher und arabischer Sprache (den Folder in deutscher Sprache finden Sie unter http://www.kontinenzgesellschaft.at; Betroffenen-Info). Der Patientenfolder in Türkisch und Arabisch wurde nur aktualisiert, etwas gekürzt und enthält zusätzlich ein klares Statement der IGGÖ betreffend die Voraussetzungen, unter denen Muslime trotz Inkontinenz ihre religiösen Pflichten ausüben dürfen.

www.kontinenzgesellschaft.at 